\title{
An alternative analysis of contrast-variation neutron scattering data of casein micelles in semi-deuterated milk
}

\author{
Gregory N. Smith
}

\section{Published version information}

Citation: GN Smith. 'An alternative analysis of contrast-variation neutron scattering data of casein micelles in semi-deuterated milk.' European Physical Journal E, vol. 44, no. 1 (2021): 5.

DOI: $10.1140 /$ epje/s10189-021-00023-y

This is a post peer-review, pre-copyedit version of an article published in EPJ E. The final authenticated version is available online at the DOI above.

This version is made available in accordance with publisher policies. Please cite only the published version using the reference above. This is the citation assigned by the publisher at the time of issuing the AAM. Please check the publisher's website for any updates. 


\title{
An alternative analysis of contrast-variation neutron scattering data of casein micelles in semi-deuterated milk
}

\author{
Gregory N Smith ${ }^{12}$ \\ 1 Niels Bohr Institute, University of Copenhagen, Universitetsparken 5, 2100 Copenhagen Ø, Denmark \\ 2 ISIS Neutron and Muon Source, Science and Technology Facilities Council, Rutherford Appleton Laboratory, Didcot, OX11 \\ 0QX, United Kingdom, gregory.smith@stfc.ac.uk
}

Received: date / Revised version: date

\begin{abstract}
Contrast-variation small-angle neutron scattering (CV-SANS) is an excellent way to determine the structure of complex, hierarchical colloids, including self-assembled biological systems. In these experiments, the scattering length density of solvents is changed (by varying the ratio of water or $\mathrm{H}_{2} \mathrm{O}$ and heavy water or $\mathrm{D}_{2} \mathrm{O}$ ) to highlight or mask scattering from different components in the system. This approach has been used with synthetic colloids, but it is also increasingly being used in the biological and food sciences. Perhaps the most studied food colloid is the "casein micelle", a self-assembled nanometer-scale colloid of the structure-forming casein protein in milk. CV-SANS data available in the literature are typically analyzed using approximations, which may be invalid for casein micelles, as they have been shown to be sticky spheres. To assess the applicability of this approximate approach, a comprehensive set of CV-SANS data from casein micelles in diluted milk was reanalyzed using a model-based approach, where the casein micelles were formally treated as interacting spheres. In general, the conclusions of the previous study are reproduced, but this new approach makes it more straightforward to distinguish the different components in milk and can be applied to any dairy sample with known form of interparticle interactions, which offers the possibility of studying semi-deuterated milk at its native concentration.
\end{abstract}

PACS. XX.XX.XX No PACS code given

\section{Introduction}

Neutron methods are increasingly finding application as powerful ways of studying biological systems [1-3]. This includes various examples, such as determining structures of biological molecules in solution, the structures of protein crystals, the dynamics of protein molecules in the field of structural biology, the structure of biological membranes, or imaging of entire organisms [2,3]. The specific sensitivity of neutrons for the stable isotopes of hydrogen that are encountered in biological materials $\left({ }^{1} \mathrm{H}\right.$ protium and ${ }^{2} \mathrm{H}$ deuterium [4-8]) is what makes these many experiments possible [9-12], particularly those where neutrons provide information that cannot be obtained using other techniques. The example applications of neutron techniques to biophysics and biochemistry experiments given above $[2,3]$ are typically on pure biological materials, but neutron techniques are also increasingly finding use in more applied areas of the biosciences, including in the area of food science [13-15].

The increase in isotopic labeling of biomolecules in recent decades [5-8] is what enables these experiments in food science. Neutron techniques are applied to biological systems for many purposes $[2,3]$, but there are two

Send offprint requests to: primary opportunities for neutron experiments that arise from the difference between protium and deuterium nuclei. One arises from the fact that the incoherent scattering cross section of ${ }^{1} \mathrm{H}$ is much higher than ${ }^{2} \mathrm{H}$ [16], and this makes it possible to differentiate between the dynamics of hydrogen nuclei that are differently labeled in an inelastic neutron scattering experiment. Such experiments have been performed on food-relevant systems like proteins [17], saccharides [18-20], vitamins [21], and even on food products such as fruits and vegetables [22] or bread [23]. The other arises from the fact that the coherent cross section of ${ }^{1} \mathrm{H}$ and ${ }^{2} \mathrm{H}$ are very different [16], and this means that selectively deuterating either the solvent or the dispersed phase makes it possible to determine the distribution of species. Such experiments have been performed on foodrelevant systems like bile salt micelles [24,25], oleosomes [26], starch [27], and even on deuterated alcoholic beverages $[28,29]$. The latter is the particular approach to neutron scattering that is employed in this study, which is particularly powerful for molecular biology and food science. This approach is called "contrast variation" where many measurements are performed on isotopically distinguishable but chemically identical species, where the light (protium, ${ }^{1} \mathrm{H}$ ) is exchanged for the heavy (deuterium, ${ }^{2} \mathrm{H}$ ) isotopes of hydrogen [30,31]. 
Milk is a ubiquitous and important biological food material that is interesting to study from a biophysical or biochemical approach. This is due to its complex structure and the changes that it can undergo during processing to produce a variety of food products [32]. Structural changes are key to enabling the production of these dairy products, and in this context, it is the structure-forming protein that is the important one to study. Casein protein forms socalled "casein micelles" in solution, which are protein colloids with a complex and hierarchical structure. The importance of these colloids has resulted in a long-standing effort to understand their internal structure, and this has been well reviewed in recent years and so will not be exhaustively discussed here [33-35]. A specific approach that has been very insightful is the application of contrastvariation small-angle neutron scattering (CV-SANS), introduced above. This is because casein micelles have a hierarchical, complex, and multi-component structure and because they are highly hydrated. There are already several reports in the literature of milk prepared in either wholly or partially deuterated water [36-44,34, 45, 46]. Different approaches to modeling the contrast variation data have been used, but several employ [36,45] an approach using the Guinier approximation [47] that relates the radius of gyration $\left(R_{g}\right)$ to the reciprocal difference in scattering contrast $(1 / \bar{\rho})$, popularized by Stuhrmann [48]. This approach can be used to reveal the homogeneity or heterogeneity and the distribution of scattering contrast from a consideration of the scattering at very low $Q$. It has proved insightful in the study of biomolecules in solution [49-51] and in colloid science generally [52-55].

The Stuhrmann analysis is an elegant way to use SANS to study how homogenous a biomolecule or colloid is, but it must be used with care, due its basis on the Guinier approximation [47]. The Guinier approximation must be valid, for the Stuhrmann approach to be valid. The Guinier approximation has several requirements. It is only appropriate when the product of $Q$ and the particle gyration radius $r$ is much less than 1 [47,52], when particles are noninteracting $[10,56]$, and when there is only one population of scattering object present. To achieve this, particularly near the average contrast-match point, background subtraction and absolute intensity calibration must be excellent. These points are the most important for the Stuhrmann analysis, but they are also the points where this is most challenging in practice. For casein micelles at a concentration in normal milk [57], the Guinier approximation would be inappropriate. Recent ultra-smallangle X-ray scattering data showed that casein micelles in skim milk cannot be treated as dilute or noninteracting spheres. To successfully model the data, they must be treated as sticky spheres [58]. The applicability of this model was then tested with neutron scattering data as well (both ultra-small-angle neutron scattering and spinecho small-angle neutron scattering) and was found to provide improved agreement between experimental data and model calculation [59]. The scattering at low $Q$, where the Guinier approximation is applied, depends on the specific

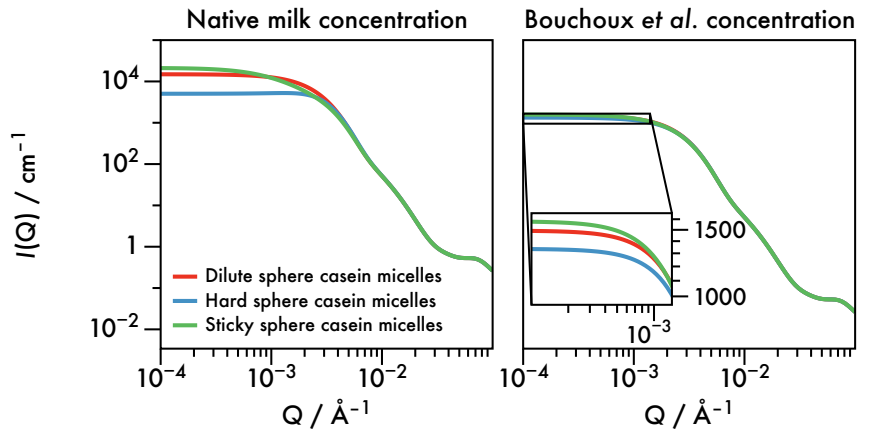

Fig. 1. Simulated X-ray scattering intensity to show the predicted scattering from casein micelles for different degrees of interaction (dilute sphere, hard sphere, or sticky sphere) between the casein micelle colloids. Other than the interactions between spheres, the casein micelles have the same dimensions. (Values come from Smith et al. [58].) At native milk concentrations, the scattering differs significantly for these three interactions. For the diluted dispersions used in the study by Bouchoux et al. [45], the scattering is much more similar for these three interactions. However, as can be seen by the low $Q$ magnification shown in the inset, the predicted scattering is not equal. This shows that the Stuhrmann analysis is more valid than at normal milk concentrations, yet not completely.

interparticle interactions and may differ from that of dilute particles.

Fig. 1 shows calculated, simulated scattering X-ray scattering intensity using the parameters from the literature [58], and it is immediately apparent that the real dispersions of casein micelles (sticky sphere casein micelles) are different than the dispersions that would be necessary to apply the Guinier approximation (dilute sphere casein micelles). For diluted dispersions of casein micelles $(10 \times$ more dilute as in Bouchoux et al. [45]), the calculated, simulated scattering (also shown in fig. 1) shows little difference between different structure factors, at first glance. However, even in these diluted dispersions, the scattering from casein micelles as dilute spheres, hard spheres, and sticky spheres differ. (The data are shown on logarithmic axes, so what appear to be small differences are in fact significant.) This shows that these dilute dispersions are more suitable for the requirements of the Guinier approximation (regarding the degree of particle interactions) than at native milk concentration, but the application of this approximation is still not ideal.

In recent years, several publications have reported contrast-variation SANS data of casein micelles at relatively low $Q[45,46]$. Here, a reanalysis was performed on one of these sets of data, that of Bouchoux et al. [45]. The original publication presents a comprehensive set of data of the scattering of casein micelles in a large number of solvent contrasts. However, unlike in the original publication, the Stuhrmann and Guinier approach $[47,48]$ was used, and a full model fitting approach was used instead. This is partly motivated by several observations from the original publication (non-zero scattering when the solvent is matched to the average contrast of the casein micelles and the presence of a second population of objects with 
a different contrast) and by a desire to ensure that this approximate approach can match the results of a more complicated approach.

This is the same motivation given in a classical study of colloidal science from Ottewill, where a curve-fitting and a Guinier approach were both performed as the "more complete analysis, however, validates the [Guinier] approach as a relatively quick method of obtaining information ... and demonstrates the interesting scattering behaviour shown by these particles in the vicinity of the contrast match point" [60]. The comparison between the Guinier approach and a model-fitting approach for a archetypal food colloid reveals when an approximation can be applied, when model-fitting must be used, and also when a small-angle scattering $Q$ range is valid and when an ultrasmall-angle $Q$ range is necessary. Further, model-fitting analysis will be more versatile, as it could be used for contrast-variation studies of any deuterated milk samples, not just highly diluted ones.

\section{Data and Analysis}

\subsection{Scattering data}

The FM_s1 data set from Bouchoux et al. is used for analysis here. The neutron scattering data were obtained from the low momentum transfer and low background SANS instrument D11 at the Institut Laue-Langevin (ILL) in Grenoble, France [61-63]. D11 has a very long maximum sample-to-detector distance of $39 \mathrm{~m}$, and depending on the neutron wavelength $(\lambda)$ selected, this makes it possible to reach very low values of the momentum transfer vector, $\boldsymbol{Q}$. The magnitude of $\boldsymbol{Q}$ is determined by the scattering angle $(\theta$, which is half the scattering angle) and $\lambda[10]$.

$$
Q=\frac{4 \pi \sin \theta}{\lambda}
$$

The specific configuration used for the experiments is provided in the original publication [45]. The authors obtained data over the range $9 \times 10^{-4}<Q<3 \times 10^{-2}$ $\AA^{-1}$. Uncertainties for the data are not shown, but they are available in the original publication. For high-contrast samples, the uncertainties are, in general, smaller than the size of the data point in the original publication $(\Delta I / I \lesssim$ $15 \%)$. For low-contrast samples, the errors, particularly at extremes in $Q$, can be large $(\Delta I / I \lesssim 80 \%)$, but this can be appreciated in the data presented here without the inclusion of error bars, as the data are consequently noisy.

The data were obtained from publications using the macOS application GraphClick [64], which has been shown to be a reliable and viable method to extract data from images.

\subsection{Model fitting}

The data are modeled using a multilevel model as described in the text using the SASfit (version 0.94.11) fitting software [65]. The data were fit as a sum of two contributions. The choice of these are discussed more in the text. Due to the lack of clarity on the uncertainties, which would all be equal at $\lesssim 15 \%$ when smaller than the size of the data point, the data were fit without using error bars. The weighting of the fit sought to achieve local smoothness.

The major contribution to the scattering intensity in the measured $Q$ range is the scattering of the casein micelles. These are modeled as homogeneous spheres with a log-normal distribution in their radius. The spherical form factor depends only on the radius $(r)$ of the spheres [66].

$$
P(Q)=\left[\frac{3(\sin (Q r)-Q r \cos (Q r))}{(Q r)^{3}}\right]^{2}
$$

The distribution of sizes is given by the log-normal function, where the logarithm of a variable (in this case the radius) has a normal distribution. The function is given elsewhere [67].

The spheres are assumed to have a nonrandom distribution in the dispersion, and their interparticle interactions are accounted for using a sticky sphere structure factor $(S(Q))$. As far as I am aware, no X-ray or neutron scattering data in the literature have required accounting for specific interparticle interactions to fit the data [58], although de Kruif and May did attempt fitting neutron scattering data of $\kappa$-casein micelles using different structure factors, but the results were inconclusive [68]. In my previous analysis of USAXS data from skim milk [58], Baxter's model of adhesive spheres was used [69]. This model was favored because the magnitude of the attraction is quantified in a single parameter as discussed below, and this will enable computational simplicity and reduce the number of fitting parameters. There is a range of interparticle separations $(\Delta)$ where the interaction potential is negative. In the Baxter model [69] of adhesive spheres, the pair interaction potential $U(h)$ is given by eq. 3 (in units of the thermal energy, $k_{B} T$ ).

$$
U(h) / k_{B} T=\left\{\begin{array}{cll}
\infty & \text { for } & h<2 r \\
\ln \left(\frac{12 \tau \Delta}{\sigma+\Delta}\right) & \text { for } & 2 r<h<2 r+\Delta \\
0 & \text { for } & h>2 r+\Delta
\end{array}\right.
$$

The attraction is then calculated in the limit $\Delta \rightarrow 0$ [67]. This results in a well that is infinitely deep but infinitesimally narrow [70]. The adhesive strength is, therefore, encapsulated in a single parameter called $\tau$, the stickiness parameter. This can be thought of a dimensionless measurement of the temperature [69] and can be used to calculate the second virial coefficient, enabling a comparison with theory and other experiments [71]. The sticky sphere model has shown to be valuable in studying a variety of interacting systems and has, therefore, been much considered in the literature [72]. This sticky sphere model has been used to determine the equation of state experimentally [73] and theoretically [74]. For the volume fraction and stickiness parameter found for casein micelle dispersions in skim milk [58], the dispersions are in the nonpercolating fluid part of the phase diagram $[75,74,76]$. However, that may not always the case for other casein micelle dispersions or in treated milk, and the use of this structure 
factor provides a way of relating colloidal interparticle interactions to the phase behavior.

The size distribution is accounted for using the local monodisperse approximation, where particles are assumed to always be surrounded by particles of the same size and the scattering scaled according to the size distribution [77]. The local mononodisperse approximation was favored over other ways of accounting for the size distribution (specifically, the monodisperse approach or the decoupling approach) as it is known to work better for systems with broad size distributions and higher concentrations [67]. The effect of concentration, size distribution, and stickiness on the structure factor is known to result in complex behavior [78], and the selection of different structure factors or approaches to incorporating the size distribution would impact the best fit values to this model. After careful consideration, the model selected previously was found to give the best agreement between the data and the calculated scattering [58].

The data presented here are fit to a spherical model, but ultra-small-angle scattering data in the literature [7983] have alternatively been fit using Beaucage's unified approach $[84,85]$. This model calculates scattering as a summation of multiple levels, each consisting of a Guinier function (with a radius of gyration) and a Porod function (with a power law exponent). There is no straightforward way of accounting for interparticle interactions using a structure factor in the Beaucage approach. The radii of gyration of casein micelles found using this approach in the literature have sphere equivalent radii that are greater than fit using the spherical modeling approach $[86,58]$. The two approaches give fits to the data of similar quality; for example, the data of Pignon et al. are fit to the Beaucage model originally [79] but can also be fit using the sphere model proposed recently [58] (Supporting Information). Due to the ability to directly extract quantitative parameters regarding interparticle interactions, the spherical model is preferred for the analysis discussed here.

The minor contribution to the scattering intensity in this $Q$ range is the interface of large droplets. If it is assumed that they have a sharp interface, then in the socalled Porod limit $[47,87]$, scattering decreases as a power law in $Q$ with a power law index of -4 . In the limit as $Q$ goes to infinity, the scattering intensity depends on the specific area $(\Sigma)$ of the sample and contrast between particle and medium $(\Delta \rho)$, although to calculate this accurately necessitates very good background subtraction and intensity normalization [10].

$$
\lim I(Q)_{Q \rightarrow \infty}=\frac{2 \pi\left(\Delta \rho^{2}\right) \Sigma}{Q^{4}}
$$

\section{Results and Discussion}

The Stuhrmann approach [48] can be used with great success as a simple way to analyze contrast-variation SANS data, and this has been done with diluted dispersions of casein micelles $[36,45]$. One of these sets of data (that of Bouchoux et al. [45]) is the one that has been reanalyzed
Table 1. Best fit parameters for casein micelles as sticky spheres from Smith et al. [58].

\begin{tabular}{cc}
\hline Form factor, $P(Q)$ & \\
\hline Number density, $N^{\ddagger} /\left(10^{20} \mathrm{~m}^{-3}\right)$ & $0.198 \pm 0.005$ \\
Median sphere radius, $r / \AA$ & $478 \pm 7$ \\
Log-normal distribution width, $\sigma$ & $0.311 \pm 0.006$ \\
Volume fraction, $\phi^{\ddagger}$ & $0.0140 \pm 0.0005$ \\
\hline \hline Structure factor, $S(Q)$ & \\
\hline Stickiness parameter, $\tau$ & $0.165 \pm 0.002$ \\
Volume fraction, $\phi^{* \ddagger}$ & $0.01400 \pm 0.00009$ \\
\hline
\end{tabular}

${ }^{\ddagger}$ Reduced by $10 \times$ to correct for concentration.

in this study. Rather than using the Stuhrmann approach as done in the original report [45], however, I have used a model-based approach based on recent data that showed that casein micelles in milk are sticky spheres [58]. This approach can be applied to contrast-variation SANS data at any concentration, as it does not require the Guinier approximation to be valid. The data chosen for reanalysis [45] has a large number of solvent contrasts, which makes it suited to reconsideration but is not the only report of contrast variation SANS experiments of semi-deuterated milk in the literature $[36-44,34,45,46]$.

The $Q$ range of SANS data is insufficient to fit the four parameter model proposed to cover the entire structural hierarchy of the casein micelle $[58,86]$ with certainty, as the smallest three contributions do not dominate the scattering over this $Q$ range. (The four parameters are, from largest size to smallest size, the casein micelle colloids, sponge-like incompressible regions of protein, colloidal calcium phosphate, and protein inhomogeneities.) Therefore, dimensions for the casein micelles determined from the fits to literature data are used instead [58]. The best fit parameters are shown in table 1 and are set to equal to literature values, except for the concentration of casein micelles, which are $10 \times$ less for the current data. These values were fit from data taken over a broad $Q$ range, but they come from fitting a single casein micelle dispersion at a single set of conditions. Performing this same analysis on a series of dispersions with different number concentrations of casein micelles and tuned interactions would add further certainty to the parameters. Furthermore, while every attempt was made to reduce the number of fitting parameters [58] to reduce uncertainties in fitting, some parameters are still correlated (as can be determined from the covariance matrix). Specifically, the stickiness parameter $\tau$ is correlated most with the other fitting parameters from the casein micelle scattering and is most correlated with the width of the log-normal size distribution. To account for residual scattering from larger species present in the dispersions, which may dominate as the scattering length density (SLD, $\rho$ ) of the medium approaches that of casein protein, a power law $\left(\propto Q^{-4}\right)$ is also added to fit the data.

As the concentration and structural dimensions of the casein micelles are fixed (table 1), the model is highly con- 
strained. There are only two parameters that are allowed to vary: the difference in SLD between the medium and the casein micelles $(\Delta \rho)$ and the scale of the $Q^{4}$ power law $\left(c_{4}\right)$. This is a highly constrained model, but as the $Q$ range of the data is less than an order of magnitude, to allow too many parameters to vary would risk overfitting the data. Fig. 2 shows the experimental data of Bouchoux et al. [45] (with the solvent SLD shown in the legends along with the proportion of $\mathrm{D}_{2} \mathrm{O}$ in the medium) along with the new fits using the model described. There is extremely good agreement between the data and the model, which suggests that this highly constrained model is a good description of the data.

The two contributions to the scattering data arise from different components in the milk, and so the degree that each contributes will differ depending on the solvent SLD. At the most extreme solvent SLDs, when either the proportion of $\mathrm{H}_{2} \mathrm{O}$ or $\mathrm{D}_{2} \mathrm{O}$ is high, the casein micelles contribute significantly as they are the species with the most feature-rich scattering in the measured $Q$ range. When the SLD of the solvent is $\sim 45 \% \mathrm{D}_{2} \mathrm{O}$, the SLD of the solvent is nearly the same as the SLD of the casein protein, and the significance of the scattering from the $Q^{-4}$ power law is greatest. This can be seen in fig. 2 when the total fits to the data (solid lines) are most different to the fit to the casein micelles alone (dashed lines). From the fits, it will be possible to determine at what point both contributions are contrast matched to the solvent.

To quantify the contrast between two contributions and the medium, different approaches are used. For the casein micelles, where the structural parameters are known from the literature [58], fitting the scattering intensity gives $\Delta \rho^{2}$ directly, as the concentration of particles and their volumes are specified during the fitting. For the large objects, their radius cannot be fit with certainty as it beyond the minimum $Q$ of the measurements. The scattering in the measured $Q$ range, therefore, represents the smooth interface of the large objects. The large objects could, potentially, be characterized in the same manner as the casein micelles if measurements at lower $Q$ were performed, such as by using ultra-small-angle scattering techniques. Fig. 3 shows an extrapolation of the scattering of the large objects (dashed lines) to the approximate minimum $Q$ attainable by typical ultra-small-angle scattering instruments $\left(10^{-5} \AA^{-1}\right)$. The degree that the power law scattering contributes to the total scattering intensity depends on the solvent SLD, as expected. The inset in the middle panel of fig. 3 shows the calculated scattering from the large objects determined from ultra-small-angle scattering data by Adams et al. [46] compared to the fit $Q^{-4}$ power law. Over the $Q$ range of these SANS measurements (boundaries in green), the two are equal, and this shows that these data cannot be used to determine the size with any certainty. Therefore, the fit scale of the power law $\left(c_{4}\right)$ is used as a proxy for $\Delta \rho^{2}$. This makes the assumption that the particles are the same size and at the same concentration, and then the only difference between the different solvent media is the SLD difference.
For dispersions of homogeneous and isotropic scatterers, the scattering intensity $(I(Q))$ is given by several scaling factors and $Q$-dependent functions [10], where $\phi$ is the volume fraction, $V_{p}$ is the volume of the particle, $\Delta \rho$ is the SLD difference between the dispersed phase and the solvent, $P(Q)$ is the form factor, and $S(Q)$ is the structure factor.

$$
I(Q)=\phi V_{p} \Delta \rho^{2} P(Q) S(Q)
$$

For both contributions to the scattering (casein micelles and large objects), the particles are assumed to be identical at all solvent compositions. This means that every parameter in eq. 5 is equivalent, except for the difference in SLD, $\Delta \rho$. Therefore, either a plot of the fit SLD difference $(\Delta \rho)$ against the solvent SLD (in the case of the casein micelles where the scattering curve is fit) or a plot of the square root of the $c_{4}$ scale $\left(\sqrt{c_{4}}\right)$ against the solvent SLD (in the case of the large objects where the radius is unknown) will meet the abscissa at the SLD of the dispersed phase. (At this point, $\Delta \rho=0$ ). Fig. 4 shows these plots. Similar plots are obtained for both contributions. The contrast terms $\left(\Delta \rho\right.$ or $\left.\sqrt{c_{4}}\right)$ are an absolute value function of the solvent $\operatorname{SLD}(\rho)$. The function is fit weighted by the reciprocal square of the uncertainty from fitting.

The point where $\Delta \rho$ is equal to 0 for the casein micelles is at an SLD of $2.49 \times 10^{-6} \AA^{-1}$, and the point where $\Delta \rho$ is equal to 0 for the large is at an SLD of $0.28 \times 10^{-6} \AA^{-1}$. This compares to values determined by Bouchoux et al. [45] of $2.43 \times 10^{-6} \AA^{-1}$ for the casein micelles and $0.13 \times 10^{-6} \AA^{-1}$ for the large objects. These values compare favorably, which shows that the two analyses are consistent ways of interpreting the same set of data, for determining the SLD components, at least. The value of the SLD of the casein micelles agrees well with literature values for casein $[37,88]$. The value of the SLD of the large particles is very similar to the value that would be expected for fatty acids in dairy milk. The SLD of dairy fat can be estimated by calculating that of similar triglycerides formed of the most common saturated and unsaturated fatty acids in bovine milk [89] using the high temperature mass densities from the literature [90, 91]. The calculated SLD of tripalmitin is $0.05 \times 10^{-6}$ and of triolein is $0.15 \times 10^{-6}$, which are similar in magnitude, albeit lower, than the value found from the SANS data here. Therefore, the proposal from previous neutron scattering data that these large particles are either fat globules [46] or fat droplets or phospholipids [45] seems reasonable. The quality of the determination of these values has been checked by recalculating the scattering curves in fig. 2 using the ideal values determined in fig. 4. The agreement between experimental data and model calculation is still very good, which supports the accuracy of these experimentally-determined SLDs.

\section{Conclusions}

The Stuhrmann analysis is an elegant way of analyzing contrast-variation SANS data, but the fact that it requires 

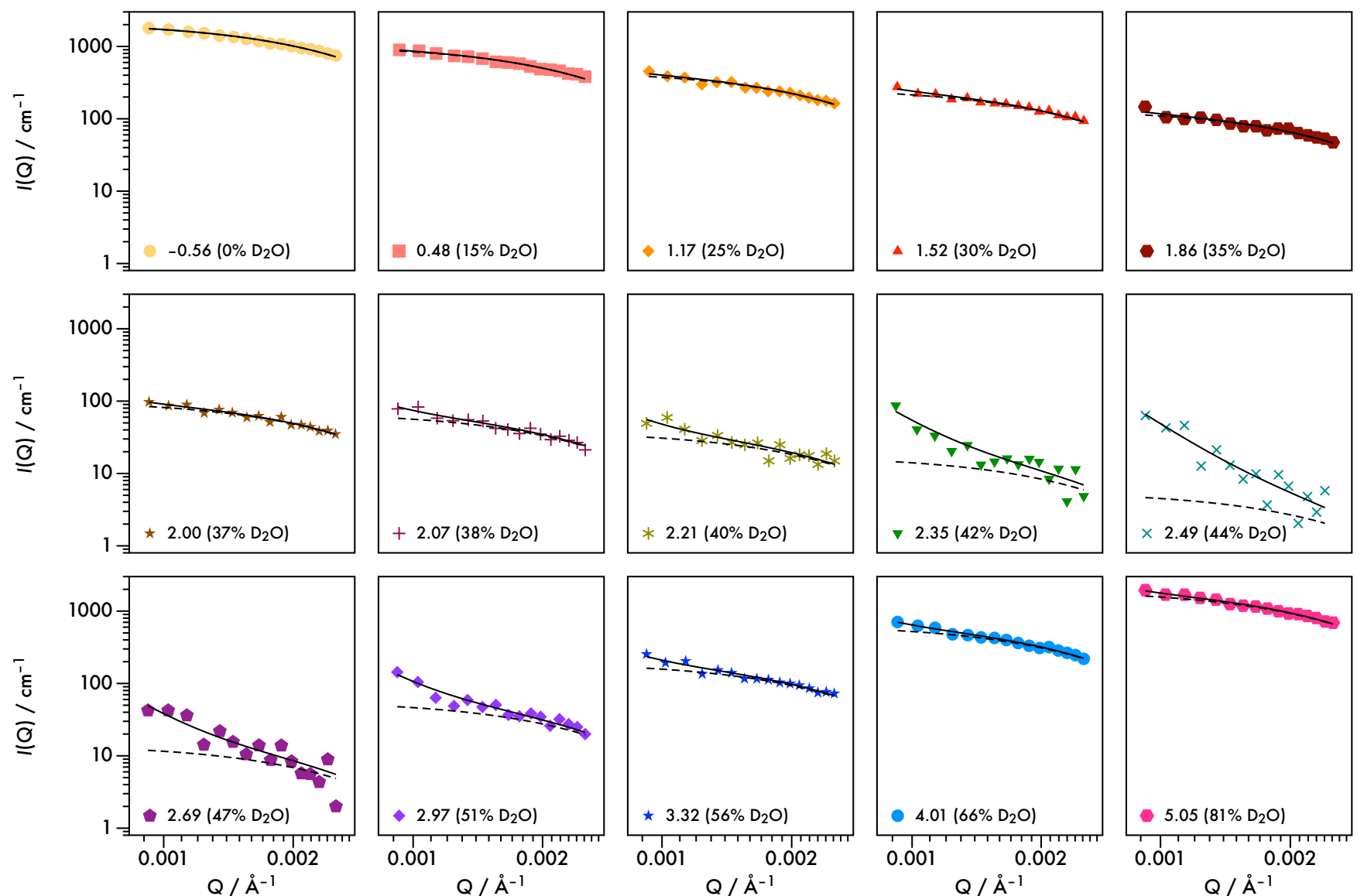

Fig. 2. The experimental data of Bouchoux et al. [45] and fits to the two contribution model described in the text (casein micelles as sticky spheres and large objects with a smooth interface). The legends for each set of data gives the SLD of the solvent medium (in units of $10^{-6} \AA^{-2}$ ) and the proportion of $\mathrm{D}_{2} \mathrm{O}$ in the mixture. The solid lines show the sum of the scattering from both contributions, and the dashed line shows the scattering from the casein micelles alone, after subtracting the $Q^{-4}$ power law. The scattering from the casein micelles is least significant, and consequently the $Q^{-4}$ power law most significant, for solvents where the proportion of $\mathrm{D}_{2} \mathrm{O}$ is $\sim 45 \%$, which have SLDs nearly the same as the casein protein.
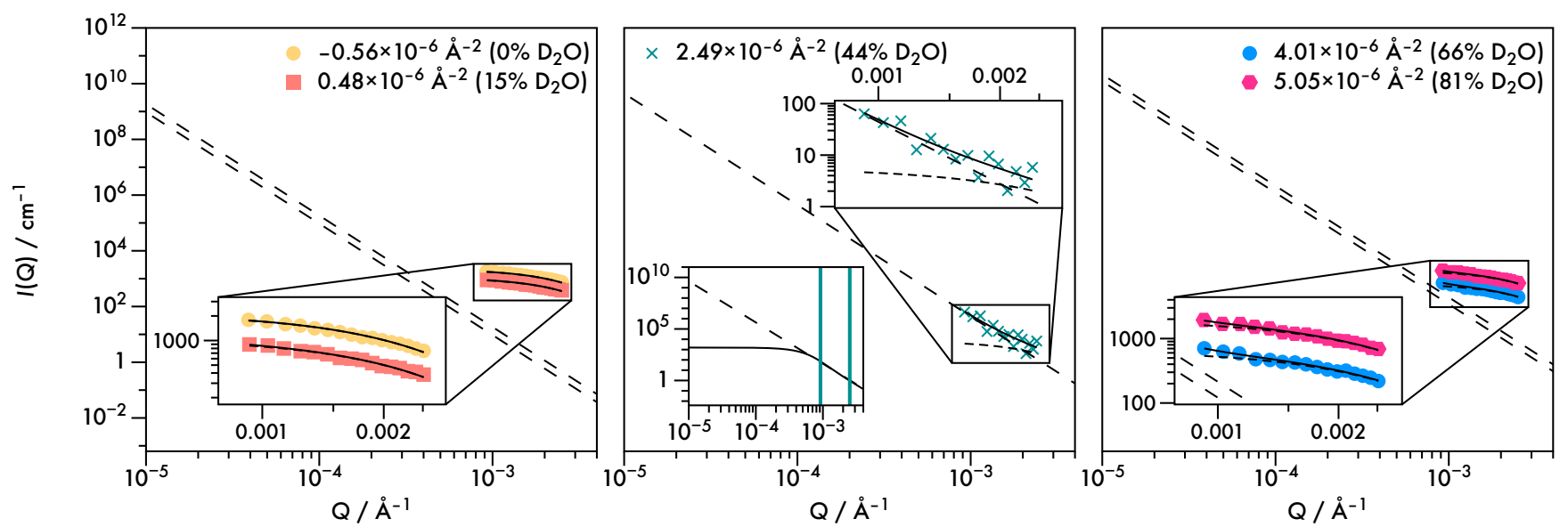

Fig. 3. Selected SANS fits (solvents given in the legends) from fig. 2 with the $Q^{-4}$ power law extrapolated to an ultra-smallangle scattering minimum $Q$ of $10^{-5} \AA^{-1}$. The significance of the power law scattering is least at extreme proportions of either $\mathrm{H}_{2} \mathrm{O}$ or $\mathrm{D}_{2} \mathrm{O}$ (left and right), although they are more significant when the proportion of $\mathrm{D}_{2} \mathrm{O}$ is high, which suggests that the SLD of the large objects is closer to the SLD of $\mathrm{H}_{2} \mathrm{O}$. The significance of the power law scattering to the total scattering is greatest when the fit $\Delta \rho$ for the casein micelles is lowest (middle). The inset in the middle panel shows the $Q^{-4}$ power law compared to the calculated scattering from large droplets with radii determined by Adams et al. [46], which shows that in the $Q$ range of these measurements, the radius of the objects cannot be determined with certainty. 

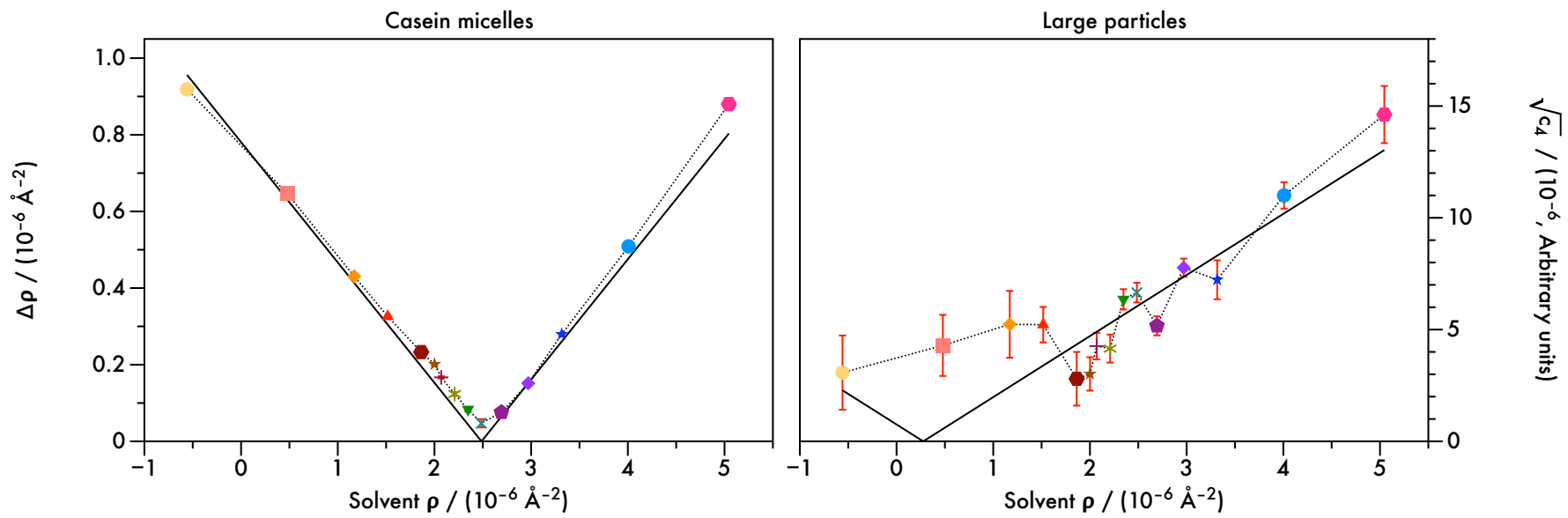

Fig. 4. The contrast terms from fitting SANS data at all solvent compositions (fig. 2) used to determine the SLD of both the casein micelles (left) and the large particles (right). For the casein micelles, the difference in SLD $(\Delta \rho)$ from model fitting is used. For the large particles, the scale of the $Q^{-4}$ power law is used, as they are assumed to have the same size regardless of the solvent composition. The point where the fit meets the abscissa is when the forward scattering intensity is 0, and the SLD of the particles is equal to that of the solvent.

using the Guinier approximation means that there are limitations to its application. Dispersions need to be dilute, particles need to be noninteracting, and only one scattering species can be present. The casein micelles in milk were recently shown to be sticky spheres rather than dilute spheres [58], and even in skim milk, there may be residual fat droplets or other lipids as well as the casein micelles $[45,46]$. Even when diluted, these issues may still remain. This motivated a careful reanalysis of an extensive set of contrast-variation SANS data from milk diluted with solvents with different proportions of $\mathrm{H}_{2} \mathrm{O}$ and $\mathrm{D}_{2} \mathrm{O}$ [45], to vary the contrast between the different species and the medium. Previously, these had been analyzed using the Stuhrmann approach. In this paper, a more general model fitting approach has been used. The scattering data were fit as a sum of two species (casein micelle colloids and large fat droplet interfaces) rather than extrapolated using the Guinier approach.

Some of the outcomes using both of these analyses are the same. The contrasts between casein micelles and the medium and the fat droplets and the medium evolve differently, showing that they are chemically distinct, and the experimentally determined SLDs of the two (when $\Delta \rho$ is 0 ) are essentially the same. This validates the values determined.

The model fitting approach has several advantages that make it more general and, therefore, preferable in some circumstances. By fitting the SANS data directly, the scattering of the two species (casein micelles and fat droplets) can be separated directly in the data, rather than having to separate them through sample preparation and then measuring the two independently. The Guinier approach assumes that the scattering arises from a single species and so cannot achieve this by data analysis. It also enables any milk sample to be studied; there is no requirement to dilute the dispersion so the colloids are dilute and noninteracting. Given that casein micelles appear to be sticky spheres, the degree of dilution necessary to make a sample suitably "noninteracting" may be hard to achieve in practice. The interactions between casein micelles are undesirable as they prevent the application of simple approximations. However, they provide the opportunity to controllably tune the interactions between casein micelles to both further validate the recently proposed sticky sphere model [58]. The ability to access thermodynamic parameters that can be related to phase behavior might enable a better understanding of milk and processed dairy products. Given that previous analyses of dispersions of selfassembled, casein-containing species found that data were sometimes fit better with repulsion and sometimes with attraction [68], there are likely circumstances where the interparticle interactions will differ, and quantifying these interactions through scattering data will aid understanding the structure and properties of dairy products. Using this model fitting approach to study a wider range of milk samples, either at native concentration or following processing, in the future using CV-SANS will reveal more about the fine details of casein micelles and milk. This may also necessitate performing measurements to lower $Q$, using a technique such as ultra-small-angle neutron scattering (USANS), to better isolate the scattering of different species, and such measurements with desmeared CV-USANS/CV-SANS data of a large number of solvent compositions would be welcome to improve data certainty. This will provide useful information about milk dispersions and casein micelles, specifically, but it will also serve as a guide to design experiments that can characterize the structure of complex food dispersions more generally.

\section{Data Availability}

The SANS data used for this analysis can be obtained from the Zenodo repository (DOI: 10.5281/zenodo.4071784). 


\section{Acknowledgments}

While at the Niels Bohr Institute, GNS was funded by the Innovation Fund Denmark (IFD) as part of project Linking Industry to Neutrons and X-rays (LINX) under File No. 5152-00005B.

\section{References}

1. T. Narayanan, H. Wacklin, O. Konovalov, R. Lund, Crystallography Reviews 23, 160 (2017)

2. R. Ashkar, H.Z. Bilheux, H. Bordallo, R. Briber, D.J.E. Callaway, X. Cheng, X.Q. Chu, J.E. Curtis, M. Dadmun, P. Fenimore et al., Acta Crystallogr. Sect. D 74, 1129 (2018)

3. D.P. Hoogerheide, V.T. Forsyth, K.A. Brown, Phys. Today 73, 37 (2020)

4. J.F. Bunnett, R.A.Y. Jones, Pure Appl. Chem. 60, 1115 (1988)

5. Arch. Biochem. Biophys. 272, 262 (1989)

6. Eur. J. Biochem. 183, 1 (1989)

7. Biol. Chem. Hoppe-Seyler 370, 1153 (1989)

8. Biochem. J. 265, I (1990)

9. R. Pynn, Los Alamos Sci. 19, 1 (1990)

10. I. Grillo, in Soft Matter Characterization, edited by R. Borsali, R. Pecora (Springer Netherlands, 2008), pp. 723-782, ISBN 978-1-4020-4464-9

11. M.J. Hollamby, Phys. Chem. Chem. Phys. 15, 10566 (2013)

12. B. Hammouda, Probing nanoscale structures-The toolbox http://www.ncnr.nist.gov/staff/hammouda/the_SANS_tool

13. A. Lopez-Rubio, E.P. Gilbert, Trends Food Sci. Tech. 20, 576 (2009)

14. E.P. Gilbert, A. Lopez-Rubio, M.J. Gidley, Characterisation Techniques in Food Materials Science (John Wiley \& Sons, Ltd, Chichester, 2012), chap. 3, pp. 52-93

15. E.P. Gilbert, Curr. Opin. Colloid Interface Sci. 42, 55 (2019)

16. V.F. Sears, Neutron News 3, 26 (1992)

17. C. Kealley, A. Sokolova, G. Kearley, E. Kemner, M. Russina, A. Faraone, W. Hamilton, E. Gilbert, Biochimica et Biophysica Acta (BBA) - Proteins and Proteomics 1804, 34 (2010)

18. M. Di Bari, F. Cavatorta, A. Deriu, G. Albanese, Biophys. J. 81, 1190 (2001)

19. M. Di Bari, A. Deriu, G. Albanese, F. Cavatorta, Chem. Phys. 292, 333 (2003)

20. L.J. Smith, D.L. Price, Z. Chowdhuri, J.W. Brady, M.L. Saboungi, J. Chem. Phys. 120, 3527 (2004)

21. E. Bellocco, D. Barreca, G. Laganà, U. Leuzzi, F. Migliardo, R.L. Torre, G. Galli, A. Galtieri, L. Minutoli, F. Squadrito, Chem. Phys. 345, 191 (2008)

22. H. Jansson, W.S. Howells, J. Swenson, J. Phys. Chem. B 110, $13786(2006)$

23. J. Sjöström, F. Kargl, F. Fernandez-Alonso, J. Swenson, J. Phys.: Condens. Matter 19, 415119 (2007)

24. O. Rezhdo, S. Di Maio, P. Le, K.C. Littrell, R.L. Carrier, S.H. Chen, J. Colloid Interface Sci. 499, 189 (2017)

25. D. Pignol, L. Ayvazian, B. Kerfelec, P. Timmins, I. Crenon, J. Hermoso, J.C. Fontecilla-Camps, C. Chapus, J. Biol. Chem. 275, 4220 (2000)
26. B.I. Zielbauer, A.J. Jackson, S. Maurer, G. Waschatko, M. Ghebremedhin, S.E. Rogers, R.K. Heenan, L. Porcar, T.A. Vilgis, J. Colloid Interface Sci. 529, 197 (2018)

27. P.J. Jenkins, A.M. Donald, Polymer 37, 5559 (1996)

28. I. Grillo, Colloids Surf. A: Physicochem. Eng. Aspects 225, $153(2003)$

29. L. Chiappisi, I. Grillo, ACS Omega 3, 15407 (2018)

30. H.B. Stuhrmann, J. Appl. Cryst. 40, s23 (2007)

31. W.T. Heller, Acta Crystallogr. Sect. D 66, 1213 (2010)

32. T. Croguennec, R. Jeantet, P. Schuck, From Milk to Dairy Products (John Wiley \& Sons, Ltd, Hoboken, NJ, USA, 2016), chap. 1, pp. 1-63, 1st edn., ISBN 9781119296225

33. D.G. Dalgleish, Soft Matter 7, 2265 (2011)

34. C.G. de Kruif, T. Huppertz, V.S. Urban, A.V. Petukhov, Adv. Colloid Interface Sci. 171-172, 36 (2012)

35. C.G. De Kruif, J. Appl. Cryst. 47, 1479 (2014)

36. P.H. Stothart, D.J. Cebula, J. Mol. Biol. 160, 391 (1982)

37. P.H. Stothart, J. Mol. Biol. 208, 635 (1989)

38. S. Hansen, R. Bauer, S.B. Lomholt, K. Bruun Quist, J.S. Pedersen, K. Mortensen, Eur. Biophys. J. 24, 143 (1996)

39. C.G. de Kruif, R. Tuinier, C. Holt, P.A. Timmins, H.S. Rollema, Langmuir 18, 4885 (2002)

40. C. Holt, C. de Kruif, R. Tuinier, P. Timmins, Colloids and Surfaces A: Physicochemical and Engineering Aspects 213, 275 (2003)

41. R.H. Tromp, W.G. Bouwman, Food Hydrocoll. 21, 154 (2007)

42. T. Huppertz, C.G. de Kruif, Int. Dairy J. 18, 556 (2008)

43. L.F. van Heijkamp, I.M. de Schepper, M. Strobl, R.H. Tromp, J.R. Heringa, W.G. Bouwman, J. Phys. Chem. A 114, $2412(2010)$

44. A.J. Jackson, D.J. McGillivray, Chem. Commun. 47, 487 $(20 \mathrm{ll} 1)$

45. A. Bouchoux, J. Ventureira, G. Gésan-Guiziou, F. GarnierLambrouin, P. Qu, C. Pasquier, S. Pézennec, R. Schweins, B. Cabane, Soft Matter 11, 389 (2015)

46. C.P. Adams, N. Callaghan-Patrachar, F. Peyronel, J. Barker, D.A. Pink, A.G. Marangoni, Food Struct. 21, $100120(2019)$

47. A. Guinier, G. Fournet, Small-Angle Scattering of X-Rays (John Wiley \& Sons, New York, 1955)

48. H.B. Stuhrmann, J. Appl. Cryst. 7, 173 (1974)

49. H.B. Stuhrmann, R.G. Kirste, Z. Phys. Chem. 46, 247 (1965)

50. K. Ibel, H. Stuhrmann, J. Mol. Biol. 93, 255 (1975)

51. B. Jacrot, Rep. Prog. Phys. 39, 911 (1976)

52. I. Marković, R.H. Ottewill, D.J. Cebula, I. Field, J.F. Marsh, Colloid Polym. Sci. 262, 648 (1984)

53. J.A.H.M. Moonen, C.G. de Kruif, A. Vrij, S. Bantle, Colloid Polym. Sci. 266, 836 (1988)

54. R.H. Ottewill, E. Sinagra, I.P. MacDonald, J.F. Marsh, R.K. Heenan, Colloid Polym. Sci. 270, 602 (1992)

55. R. Kemp, R. Sanchez, K.J. Mutch, P. Bartlett, Langmuir 26, 6967 (2010)

56. A.V. Smirnov, I.N. Deryabin, B.A. Fedorov, J. Appl. Cryst. 48, 1089 (2015)

57. H. Lindmark-Månsson, R. Fondén, H.E. Pettersson, Int. Dairy J. 13, 409 (2003)

58. G.N. Smith, E. Brok, M.V. Christiansen, L. Ahrne, Soft Matter (2020)

59. G.N. Smith, Food Hydrocoll. 113, 106511 (2021)

60. I. Marković, R.H. Ottewill, Colloid Polym. Sci. 264, 65 (1986) 
61. K. Ibel, J. Appl. Cryst. 9, 296 (1976)

62. P. Lindner, R. May, P. Timmins, Physica B: Conden. Matter 180-181, 967 (1992)

63. P. Lindner, R. Schweins, Neutron News 21, 15 (2010)

64. M.A. Boyle, A.L. Samaha, A.M. Rodewald, A.N. Hoffmann, Comput. Hum. Behav. 29, 1023 (2013)

65. I. Breßler, J. Kohlbrecher, A.F. Thünemann, J. Appl. Cryst. 48, 1587 (2015)

66. Lord Rayleigh, Proc. R. Soc. London A 84, 25 (1910)

67. J. Kohlbrecher, User guide for the SASfit software package: A program for fitting elementary structural models to small angle scattering data (April 23, 2020), Paul Scherrer Institute, Villigen, Switzerland (2020), https://github.com/SASfit/SASfit/blob/master/doc/manual/sasfit.pdf

68. C.G. de Kruif, R.P. May, Eur. J. Biochem. 200, 431 (1991)

69. R.J. Baxter, J. Chem. Phys. 49, 2770 (1968)

70. C.G. de Kruif, J. Dairy Sci. 81, 3019 (1998)

71. C.G. de Kruif, J. Colloid Interface Sci. 185, 19 (1997)

72. G. Stell, J. Stat. Phys. 63, 1203 (1991)

73. S. Buzzaccaro, R. Rusconi, R. Piazza, Phys. Rev. Lett. 99, 098301 (2007)

74. M.A. Miller, D. Frenkel, J. Chem. Phys. 121, 535 (2004)

75. H. Verduin, J.K. Dhont, J. Colloid Interface Sci. 172, 425 (1995)

76. H.G. Ruis, P. Venema, E. van der Linden, Food Hydrocoll. 21, 545 (2007)

77. J.S. Pedersen, J. Appl. Cryst. 27, 595 (1994)

78. D. Gazzillo, A. Giacometti, J. Chem. Phys. 113, 9837 (2000)

79. F. Pignon, G. Belina, T. Narayanan, X. Paubel, A. Magnin, G. Gésan-Guiziou, J. Chem. Phys. 121, 8138 (2004)

80. S. Marchin, J.L. Putaux, F. Pignon, J. Léonil, J. Chem. Phys. 126, 045101 (2007)

81. F. Pignon, G. Belina, T. Narayanan, X. Paubel, A. Magnin, G. Gésan-Guiziou, arXiv p. arXiv:0812.0879 (2008), 0812.0879

82. D.A. Pink, F. Peyronel, B. Quinn, A.G. Marangoni, Phys. Fluids 31, 077105 (2019)

83. F. Peyronel, A.G. Marangoni, D.A. Pink, Food Res. Int. 129, 108846 (2020)

84. G. Beaucage, J. Appl. Cryst. 28, 717 (1995)

85. G. Beaucage, J. Appl. Cryst. 29, 134 (1996)

86. B. Ingham, A. Smialowska, G.D. Erlangga, L. MatiaMerino, N.M. Kirby, C. Wang, R.G. Haverkamp, A.J. Carr, Soft Matter 12, 6937 (2016)

87. G. Fournet, Bull. Soc. Fr. Minér. Crist. 74, 39 (1951)

88. Y. Efimova, A. van Well, U. Hanefeld, B. Wierczinski, W.G. Bouwman, Physica B: Conden. Matter 350, E877 (2004)

89. R. Jost, Milk and Dairy Products (American Cancer Society, 2007), ISBN 9783527306732

90. D.H. Wheeler, R.W. Riemenschneider, C.E. Sando, J. Biol. Chem. 132, 687 (1940)

91. CRC, in CRC Handbook of Chemistry and Physics, edited by W.M. Haynes (CRC Press, 2011), chap. Physical Constants of Organic Compounds, 92nd edn. 